\section{Development and Effect of a Pandemic Disaster Training Program for Healthcare Providers from Designated Hospitals for Infectious Patient}

Ms. Jiyoung Noh' ${ }^{1}$, Professor Hyun Soo Chung ${ }^{2}$, Ms. Hye Mi Jin ${ }^{1}$, Ms. Jayoung Hur ${ }^{1}$, Ms. Min Ji Kim ${ }^{1}$, Ms Chan Mi Kang ${ }^{1}, M s$ Ga Hyun Lee ${ }^{1}$

1. Center for Disaster Relief, Training, and Research, Yonsei University Severance Hospital, Seoul, South Korea

2. Department of Emergency Medicine, Yonsei University College of Medicine, Seoul, South Korea

Introduction: South Korea experienced Middle East Respiratory Syndrome (MERS) outbreak in 2015. To mitigate the threat posed by MERS, the Ministry of Health and Center for Disease Control designated hospitals to be responsible for managing any suspected or confirmed infectious patient. These hospitals receive mandatory training in managing infectious patients, but many of the trainings lack practical skills practice and pandemic preparedness exercise.

Aim: To develop and evaluate a training course designed to train healthcare providers from designated hospitals to enhance their competencies in managing emerging infectious diseases and potential outbreaks.

Methods: A two-day course was developed by the Center for Disaster Relief, Training, and Research in collaboration with the Korea Health Promotion Institute using Kern's 6-step approach. The course consisted of didactic lectures, technical skills training, tabletop simulation, and scenario-based simulation. Table-top simulation exercises consisted of cases involving a single infectious patient detected in the outpatient clinic and outbreak in the emergency department. Scenario-based simulation exercises involved managing a critically ill infectious patient in an isolated ward. A post-survey questionnaire was used to evaluate the course and assess the perception changes of the participants. All pre-to-post differences within subjects were analyzed with paired t-tests.

Results: A total of 121 healthcare providers participated in three separate courses. The competencies for pandemic preparedness knowledge, skills, and attitude improved from pre- to post-course. The differences were all statistically significant $(\mathrm{p}<0.05)$. Overall course satisfaction in average for expectation, time, delivery method, and contents were 9.5, 9.2, 9.4, and 9.2, respectively.

Discussion: There needs to be tests and exercises to recognize gaps of systems in place for pandemic preparedness. Simulation exercises are ideal tools for this purpose. Although this was only a two-day intensive course, this increased familiarity with workflows, tested the coordination of workflows between different disciplines and allowed the identification of gaps.

Prehosp Disaster Med 2019;34(Suppl. 1):s118

doi:10.1017/S1049023X19002516

Development and Initial Validation of a Stochastic Discrete Event Simulation to Assess Disaster Preparedness Mattias Lantz Cronquist MSc', Carl-Oscar Jonson PhD ${ }^{2}$, Erik Prytz PhD ${ }^{1,2}$

1. Department of Computer and Information Science, Linköping University, Linköping, Sweden
2. Center for disaster medicine and traumatology, and Department of Clinical and Experimental Medicine, Linköping University, Linköping, Sweden

Introduction: Assessing disaster preparedness in a given region is a complex problem. Current methods are often resourceintensive and may lack generalizability beyond a specific scenario. Computer-based stochastic simulations may be an additional method but would require systems that are valid, flexible, and easy to use. Emergo Train System (ETS) is an analog simulation system used for disaster preparedness assessments.

Aim: To digitalize the ETS model and develop stochastic simulation software for improved disaster preparedness assessments. Methods: A simulation software was developed in C\#. The simulation model was based on ETS. Preliminary verification and validation $(\mathrm{V} \& \mathrm{~V})$ tests were performed, including unit and integration testing, trace validation, and a comparison to a prior analog ETS disaster preparedness assessment exercise. Results: The software contains medically validated patients from ETS and is capable of automatically running disaster scenarios with stochastic variations in the injury panorama, available resources, geographical location, and other variables. It consists of two main programs: an editor where scenarios can be constructed and a simulation system to evaluate the outcome. Initial $V \& V$ testing showed that the software is reliable and internally consistent. The comparison to the analog exercise showed a general high agreement in terms of patient outcome. The analog exercise featured a train derailment with 397 injured, of which 45 patients suffered preventable death. In comparison, the computer simulation ran 100 iterations of the same scenario and indicated that a median of 41 patients (IQR 31 to 44) would suffer a preventable death.

Discussion: Stochastic simulation methods can be a powerful complement to traditional capability assessments methods. The developed simulation software can be used for both assessing emergency preparedness with some validity and as a complement to analog capability assessment exercises, both as input and to validate results. Future work includes comparing the simulation to real disaster outcomes.

Prehosp Disaster Med 2019;34(Suppl. 1):s118

doi:10.1017/S1049023X19002528

Development of a New Triage Method to Prioritize Patients Arriving at the Emergency Room Dr. Tomoaki Natsukawa, Dr. Ryo Deguchi, Dr. Naoki Akita, Dr. Sadayori Uemori, Dr. Toyokazu Miki, Dr. Noboru Kato Yodogawa Christian Hospital, Suita, Osaka, Japan

Introduction: By prioritizing emergency patients, triage facilitates the timely provision of care to the largest possible number of patients arriving at an emergency room (ER). Previous triage methods include the Canadian and Japan Triage and Acuity Scales. Since these methods sort patients into five categories, multiple patients are often categorized into the same category. Furthermore, since these scales adopt original complex algorithms to determine the triage category, triage personnel need to be very familiar with the algorithm. Hence, a simple triage method is needed to prioritize ER patients. 